OPEN ACCESS

Edited by:

Hubing Shi,

Sichuan University, China

Reviewed by:

Julie Shabto,

Emory University, United States

Enrique J. Arenas,

Vall d'Hebron Institute of Oncology

(NHIO), Spain

*Correspondence:

Haiming Wei

ustcwhm@ustc.edu.cn

Specialty section:

This article was submitted to

Cancer Immunity

and Immunotherapy,

a section of the journal

Frontiers in Oncology

Received: 11 November 2020

Accepted: 04 May 2021

Published: 21 May 2021

Citation:

Zheng $X$ and Wei H (2021)

Organ-Specific Immune-Related Adverse Events for PD-1 Antibodies

in Lung Cancer Treatment.

Front. Oncol. 11:628243. doi: 10.3389/fonc.2021.628243

\section{Organ-Specific Immune-Related Adverse Events for PD-1 Antibodies in Lung Cancer Treatment}

\author{
Xiaohu Zheng ${ }^{1,2}$ and Haiming Wei ${ }^{1,2,3 *}$ \\ 1 Division of Molecular Medicine, Hefei National Laboratory for Physical Sciences at Microscale, The CAS Key Laboratory of \\ Innate Immunity and Chronic Disease, School of Life Sciences, University of Science and Technology of China, Hefei, China, \\ 2 Institute of Immunology, University of Science and Technology of China, Hefei, China, ${ }^{3}$ Research Unit Of NK Cells, Chinese \\ Academy Of Medical Sciences, Hefei, China
}

Anti-PD-1 therapy has revolutionized the clinical treatment of lung cancer. With the increasing number of lung cancer patients being treated, there is also an increase in the number of immune-related adverse events (irAEs) being reported. These irAEs involve multiple organs and systems, mainly manifest as inflammatory side effects, and are different from the adverse events observed with traditional lung cancer treatment. These effects are often mild and treatable and reversible; however, in a few cases the side effects can be severe and lead to termination of immunotherapy. Management involves glucocorticoid-based related immunomodulators, which should be carefully prescribed to balance the efficacy and side effects of the PD-1 antibody treatment. This review will describe the characteristics and mechanisms of irAEs in specific organs, and will serve as a guide to help optimize treatment plans and improve patient outcomes.

Keywords: lung cancer, immune-related adverse events (irAE), PD-1 antibody therapy, inflammatory, side effect

\section{INTRODUCTION}

Immunocheckpoint inhibitors (ICIs), especially PD-1 antibodies, have been a revolutionary success in the clinical treatment of tumors by blocking immune checkpoints to enhance anti-tumor immune responses. Normally, immune checkpoints include PD-1, which downregulates the T-cell response and serves to protect the body from potentially damaging immune responses. Tumors can hijack the system and evade the immune system by activating immune checkpoints and suppressing the T-cell response. Thus, interference with these immune checkpoint pathways can induce an antitumor immune response and deliver therapeutic benefits in cancer patients.

Several PD-1 antibodies have been approved by the United States Food and Drug Administration. Specifically, pembrolizumab and nivolumab were approved for the treatment of metastatic non-small-cell lung cancer (NSCLC). These antibody drugs have indeed shown significant efficacy in clinical trials. Programmed cell death 1 (PD-1) is a key molecule mediating immune tolerance in the body $(1,2)$. Blocking antibodies can definitely enhance the activity of the immune system, although this often results inflammatory side effects, which are referred to as immune-related adverse events (irAEs). The presence of irAEs has been reported in retrospective clinical trials evaluating PD-1 antibodies, which mainly included pembrolizumab and nivolumab, for the treatment of NSCLC (1-4). 
Clinical trial data suggest that the irAEs produced by PD-1 antibody in lung cancer treatment involve the thyroid, lung, skin, intestinal tract, and liver. Less common are the pancreas, kidney, pituitary gland, and musculoskeletal system (Figure 1). The majority of cases are mild irAEs and Anti-PD-1 therapy can usually be continued under close monitoring. Despite the very low incidence of moderate to severe irAEs, these may be associated with a serious decline in unique organ function and quality of life (5-9). Therefore, these toxicities require early detection and appropriate management. In this review, we focus on the pathological features, potential pathogenic mechanisms, and associated outcomes of irAEs in each unique organ, which is conducive to a more rational clinical management of lung cancer patients receiving PD-1 antibody treatment.

\section{THYROID DYSFUNCTION}

\section{Clinical Characteristics}

Thyroid dysfunction is a common and clinically mild irAE and is an early event among lung cancer patients treated with PD-1 antibodies (10). Most patients with anti-PD-1 drug-induced thyroid dysfunction are asymptomatic or present with hypothyroidism, hyperthyroidism, or thyroiditis $(4,5,7-9,11-$ 14). The overall incidence rates of hypothyroidism and hyperthyroidism are $9.1 \%$ and $7.8 \%$, respectively, while thyroiditis has the lowest reported incidence (2.6\%) among PD-L1-positive NSCLC patients treated with pembrolizumab monotherapy (15). Hyperthyroidism occurs shortly after the initiation of pembrolizumab treatment and presents at median after 32 days (10). The onset of hypothyroidism occur later, at median time of 98 days. Many patients who eventually develop hypothyroidism experience a brief period of asymptomatic hyperthyroidism before the onset of the disease. Hypothyroidism may be asymptomatic or mild, and continued immunotherapy should not be precluded $(7,8,10)$.

\section{Therapeutic Management}

Clinically, patients with thyroid dysfunction are routinely given long-term thyroid hormone replacement therapy (10). Patients reporting this irAE did not experience a significant recovery of thyroid function, although none of the patients required corticosteroids, $\beta$-blocker, or methimazole therapy. Patients with abnormal thyroid function test (TFT) do not need to

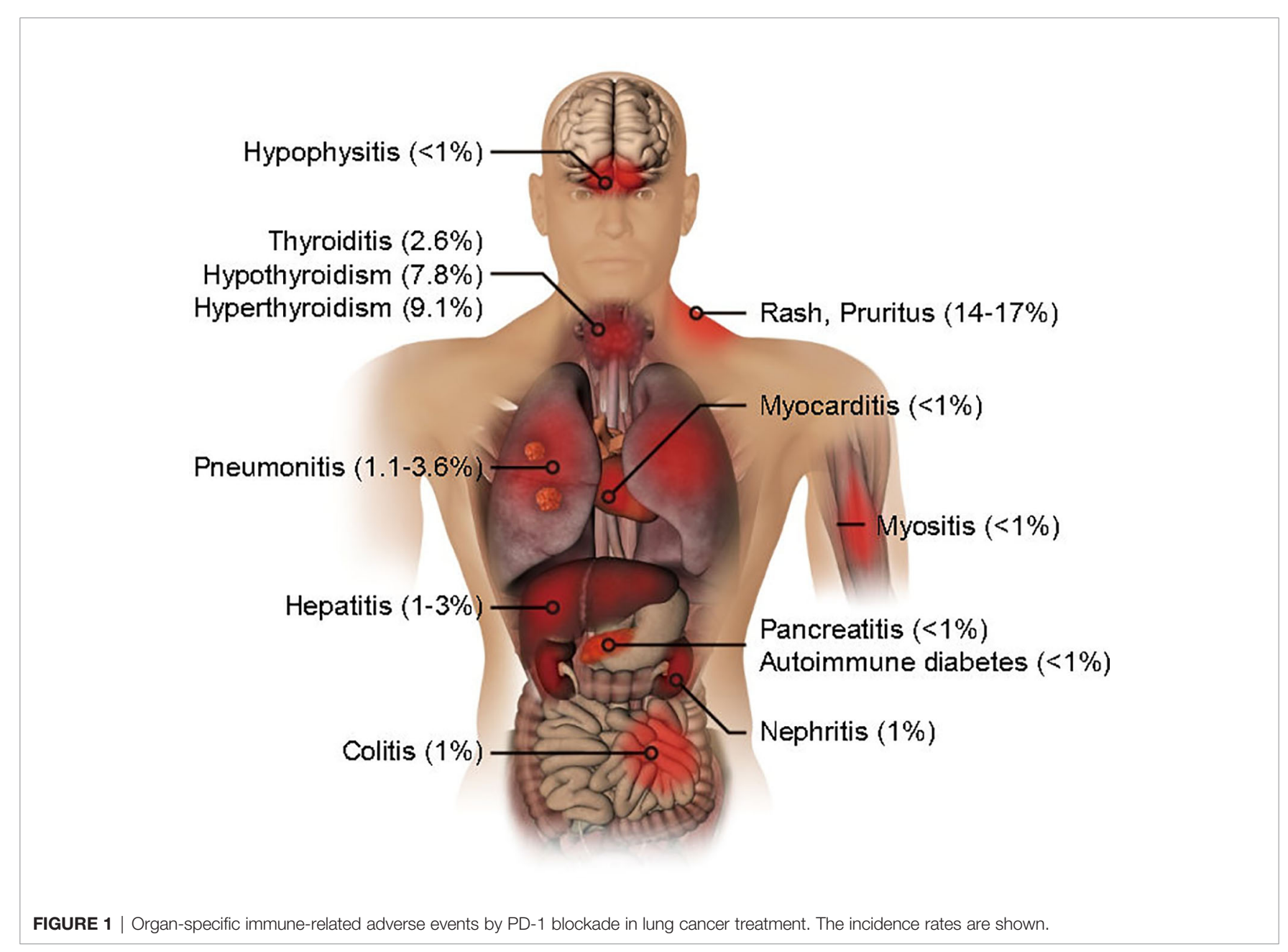


delay or stop using pembrolizumab due to the clinical impact of the thyroid dysfunction $(8,10,12,13,15)$.

\section{Association With Clinical Outcomes}

There was no significant difference in baseline clinical characteristics between patients with thyroid dysfunction and those without thyroid dysfunction. Interestingly, pembrolizumabtreated NSCLC patients with thyroid dysfunction had significantly higher median OS rates than patients without thyroid dysfunction (10). Whether there is a specific mechanistic association between antithyroid immunity and antitumor immunity is unclear, and larger clinical trials involving higher patient volumes are needed to verify the association.

\section{Possible Mechanisms/Pathophysiology}

During anti-PD-1 therapy, patients with anti-thyroid antibodies may develop thyroid dysfunction, whether or not these antibodies are present at baseline or are detected after treatment begins. In addition, many patients who eventually develop hypothyroidism experience a brief period of asymptomatic hyperthyroidism before the onset of the disease (10). In addition, to T-cell-mediated cellular immunity, anti-PD-1 therapy may also regulate humoral immunity or enhance the activity of pre-existing anti-thyroid antibodies. PD-1 plays an important role in maintaining tolerance, and Anti-PD-1 therapy may disrupt the immune system's ability to attack what it is meant to protect (16). Although it is suspected that the destruction of self-tolerance leads to thyroid autoimmunity, the mechanism through which PD-1 blocking leads to such autoimmunity is not clear.

\section{CUTANEOUS REACTIONS}

\section{Clinical Characteristics}

Dermatologic toxicity is one of the most common irAEs reported in lung cancer patients treated with PD-1 antibodies. Dermatologic toxicity manifests in a variety of forms, and commonly includes rash, pruritus, dry skin, pruritus, and dermatitis acneiform $(1,2,14$, 17). Clinically, a rash is relatively common. Specific symptoms include plaques, papules, and erythematous macules, mainly distributed to the trunk and extremities, and rashes can also be associated with pruritus (18). Dermatologic toxicity often develops in the early days following a 2-5-week treatment with anti-PD-1 blockage therapy. Dermatologic irAEs have been reported to occur in $14 \%$ to $17 \%$ of patients treated with nivolumab and pembrolizumab $(7-9,12-14,19)$. Clinically, diagnosis is usually achieved by physical examination to assess the skin appearance, while skin biopsies are performed based on the dermatologist's clinical diagnosis to define the cause (20).

\section{Therapeutic Management}

Severe skin irAEs (grade 3-5 severity, according to the CTCAE) occur in only $1-10 \%$ of lung patients receiving PD- 1 antibody therapy. Although this may vary according to clinical severity, for most patients topical corticosteroids are sufficient for treatment of rashes caused by immunotherapy (2).

\section{Association With Clinical Outcomes}

The association observed between pembrolizumab treatment and irAEs has clinical relevance because the systemic side effects of pembrolizumab can act as a proxy for therapeutic response, similar to rashes treated with EGFR tyrosine kinase inhibitors (21). A previous meta-analysis of patients with advanced melanoma who had received $\mathrm{PD}-1$ antibody immunotherapy found that the risk of death in patients with vitiligo was significantly lower than in patients without vitiligo $(22,23)$. As in the case of interleukin (IL)-2, dermatologic AEs resulting from targeted therapy are often associated with higher response rates, efficacy, and survival (24-26). Clinical data have suggested that dermatologic AEs are associated with a favorable outcomes in patients treated with pembrolizumab (27). There is still insufficient clinical data to determine whether PD-1antibody-induced irAEs are associated with a favorable outcome in lung cancer.

\section{Possible Mechanisms/Pathophysiology}

On histological evaluation, patients treated with pembrolizumab often present with an interface dermatitis or lichenoid tissue reaction. This may be due to non-specific activation of $\mathrm{T}$ cells after PD-1 blockade, resulting in attacks on susceptible keratinocytes. Ipilimumab inhibits tumor cells from evasive immune responses by suppressing the immune checkpoint cytotoxic $\mathrm{T}$ lymphocyte antigen-4 (CTLA-4), which also triggers autoimmune damage in previously protected normal cells. A similar mechanism may result for nivolumab and pembrolizumab as these antibodies target another immune checkpoint, the PD-1 receptor $(18,28-30)$.

\section{HEPATITIS}

\section{Clinical Characteristics}

Hepatitis has a prevalence of $1-3 \%$ among anti- PD-1 trials in lung cancer patients. The most common manifestation of hepatitis is an asymptomatic increase in transaminase levels, which only occurs in patients with very severe or chronic disease $(1,17)$. Monitoring of transaminase and bilirubin levels before initiation of treatment with immune checkpoint inhibitors (ICI) and after each dose is necessary for hepatitis screening. Individuals with abnormal liver enzymes should undergo additional tests to rule out viral causes or chronic diseaserelated liver dysfunction $(1,2,12,15)$. Abdominal computed tomography (CT) scans show that the severity of liver side effects varies. In mild cases, the liver appears normal. However, severe cases are characterized by hepatomegaly, weakened hepatic parenchyma, and periportal edema similar to acute hepatitis $(31,32)$.

Viral infections of the liver are a risk factor for inducing hepatitis in lung cancer patients treated with PD-1 antibodies. Patients with past exposure to a high viral load of hepatitis $B$ virus (HBV) can develop hepatitis during or after PD-1 antibody therapy. Patients with $\mathrm{HBV}$ infection can trigger more severe transaminase elevations (grade 3 or higher) (33). 


\section{Therapeutic Management and Association With Clinical Outcomes}

With the possibility of clinical therapeutic benefit and no remarkably increased risk, patients with hepatitis can choose continuous PD-1 antibody therapy. All events were of grade 3-4 and were subsequently treated with glucocorticoid or checkpoint inhibitor treatment was interrupted.

Data from case reports and phase II trials suggest that ICIs achieve a durable response and manageable safety in patients with controlled HBV or hepatitis C virus (HCV) infection (34). When treating patients presenting a history of hepatitis infection with ICI, regular monitoring of the status of the hepatitis virus is needed. Prospective studies are still needed to determine the true safety of ICI for the treatment of patients with viral hepatitis.

\section{Possible Mechanisms/Pathophysiology}

The liver is an immune-tolerant organ. PD-1 is a key molecule mediating immune tolerance of $\mathrm{T}$ cells. Any immunotherapy that blocks the PD-1 receptor is bound to break the immune tolerance microenvironment of the liver and induce hepatitis $(1,32)$. In patients with viral infection of the liver, the resting state of the virus may be disrupted, triggering viral activity and the onset of hepatitis $(32,33)$.

\section{DIARRHEA OR COLITIS}

\section{Clinical Characteristics}

For the treatment of lung cancer with PD-1 inhibitors, diarrhea is one of the most common irAEs (8-12.5\%). In contrast, colitis has been reported in $1 \%$ of patients $(1,4,5,7-9,12-14)$. The typical diagnosis of colitis includes an assessment to exclude the cause of infection and CT images to identify the severity and extent of colitis and to exclude the possibility of intestinal perforation. When a diagnosis is obscure, endoscopy is helpful. It can be used to assess patients with severe, refractory, or recurrent colitis and can help exclude cytomegalovirus-associated colitis and other high-risk characteristics $(1,35-37)$.

\section{Therapeutic Management and Association With Clinical Outcomes}

Clinicians should first exclude infectious colitis in the differential diagnosis by obtaining the patient's medical history, by examining physical appearance, or examining stool. For grade 1 symptoms, it is recommended to continue immunotherapy symptomatic treatment and close monitoring. For grade 2 symptoms, antidiarrheal use and symptomatic treatment are recommended. For persistent grade 2 symptoms, systemic corticosteroids should be attempted. If grade 3 or 4 symptoms occur, immunotherapy is discontinued with corticosteroid treatment. A monoclonal antibody against tumor necrosis factor (infliximab) is recommended for exacerbated severe symptoms and has been shown to significantly improve symptoms $(31,38-40)$.

\section{Possible Mechanisms/Pathophysiology}

The histopathological characteristics of PD-1 antibodyassociated colitis are very similar. The most common type of injury was active colitis with crypt atrophy and increased apoptosis. On biopsy, the mucosal lesions were mainly manifested as a neutrophilic crypt microabscess and inflammation, crypt atrophy, and edema. Another pattern of injury observed involved lymphocytic colitis, where biopsies showed increased intraepithelial lymphocytes (IELs), superficial epithelial injury, and increased laminar mononuclear inflammatory cells $(33,41-43)$.

\section{PNEUMONITIS}

\section{Clinical Characteristics}

The incidence of pneumonia at all levels in lung cancer was significantly higher than in other tumor types. One study reported that lung cancer patients treated with PD-1 inhibitors had significantly higher rates of full-grade interstitial lung disease (3.6\% vs. $1.3 \%)$ and advanced interstitial lung disease (1.1\% vs. $0.4 \%)$ than those treated with programmed death-ligand 1 (PDL1) inhibitors (44). Pneumonia is first determined by checking oxygen saturation whilst ambulatory, and is then confirmed by CT images, determination of the infectious agent, and the degree of inflammation. CT images exhibit variable features, including interlobular septal thickening, cryptogenic tissue, ground-glass opacity, pneumonia-like or bronchiolitis-like appearance (44). The median time from the start of treatment to the onset of pneumonia was reported to be 2.6 months. The symptoms of most pneumonia patients include cough and dyspnea $(45,46)$.

\section{Therapeutic Management}

As a clinical practice, PD-1 antibody therapy should not be terminated due to pneumonia. The vast majority of patients only need to receive corticosteroid treatment, and very few need to receive infliximab treatment. The prolonged time from the beginning of treatment to the onset of pneumonia (0.5 to 11.5 months) indicates that follow-up of signs and careful observation are important throughout therapy $(11,14,46)$.

\section{Association With Clinical Outcomes}

PD-1 inhibitor-associated pneumonia exhibits a range of imaging patterns that are associated with the level of toxicity. The safety evaluation of nivolumab in two phase I clinical trials reported pneumonitis-related death occurred in 3 cases $(2.3 \%)$ (47) and in 1 case (1.1\%) (48), respectively. The safety evaluation of pembrolizumab in two clinical trials reported pneumonitisrelated death occurred in 3 cases $(0.5 \%)$ (7) and in 1 case $(0.2 \%)$ (3), respectively. A multidisciplinary approach exploring pulmonology, radiology, oncology, and pathology is required to optimize patient care.

\section{Possible Mechanisms/Pathophysiology}

Lung cancer patients experience a higher incidence of pneumonia. The possible reasons are as follows: (1) the load of the primary lung tumor limits the stress and recovery capacity of the lung; and (2) these patients exhibit pulmonary fibrosis and chronic obstructive pulmonary disease. 


\section{NEPHRITIS}

\section{Clinical Characteristics}

In a systematic review of multiple randomized controlled trials of panitumumab and nivolumab for lung cancer, the incidence of nephritis was reported to be low (about 1\%). Elevated serum creatinine levels was the most common characteristic of renal toxicity induced by ICIs $(5,9,11,12)$. A case of interstitial nephritis was reported in the nivolumab group receiving treatment for lung cancer (9). Pauci-immune glomerulonephritis also commonly presents as a renal injury. Generally, renal injuries occur during the later stages of PD-1 antibody therapy, that is, after 6-12 months of treatment (49).

\section{Therapeutic Management and Association With Clinical Outcomes}

Most patients achieve complete relief with intravenous or oral steroid after 1-3 months. Very few patients require additional clinical hemodialysis (49).

\section{Possible Mechanisms/Pathophysiology}

Histopathologic analysis of renal biopsies from cancer patients treated with nivolumab revealed mild, diffuse, active interstitial inflammation, mild edema, and tubular epithelial injury, consisting of abundant $\mathrm{CD}^{+}$and $\mathrm{CD}^{+}{ }^{+} \mathrm{T}$ lymphocytes, and a small number of plasma cells, eosinophils and macrophages (49). In renal tissue, renal cells block the activity of $\mathrm{PD}-1$ positive $\mathrm{T}$ cells by upregulating PD-L1 expression. Therefore, when PD-1 is blocked by antibodies, the PD-1/PD-L1 signaling pathway will also be blocked, and $\mathrm{T}$ cells will further proliferate and become activated, leading to cytotoxicity and kidney injury $(49,50)$. Thus, PD-1 antibody treatment may result in nephritis as a form of altered autoimmunity, similar to how autoimmune diabetes, may be based on the loss of peripheral tolerance of reactive T cells. Any situation that leads to an increase in T cell migration and function, may cause clinically significant kidney damage $(49,51,52)$.

\section{MYOSITIS}

\section{Clinical Characteristics}

Muscle injury mainly includes myalgia and myositis, and its typical symptoms include varying degrees of muscle weakness and pain. Less than $1 \%$ of lung cancer patients treated with PD- 1 antibodies experience myositis, which is usually classified as mild (CTCAE grades 1 and 2). In general, the average onset time of myositis caused by immunotherapy is 25 days. Interestingly, ICI-associated myositis may manifest as classic muscle inflammatory symptoms, as well as ocular symptoms, similar to the autoimmune diseases observed at the neuromuscular junction (53).

\section{Therapeutic Management and Association With Clinical Outcomes}

Of particular concern is that a high percentage of myositis occurs in association with myocarditis or myasthenia gravis, both of which cause a high percentage of deaths. Therefore, clinicians need to maintain a high index of suspicion and a low threshold for skeletal muscle biopsy results. Further, more systematic heart screening is required when myocarditis occurs simultaneously $(53,54)$.

\section{Possible Mechanisms/Pathophysiology}

At present, most reports on myositis have not provided detailed clinical, immunological, and histopathological profiles, although a clinical trial study has shown that inflammation is the dominant feature and that most patients develop myositisrelated autoantibodies, such as anti-muscarinic acetylcholine receptors (mAChR) antibodies (54-56).

\section{HYPOPHYSITIS}

\section{Clinical Characteristics}

Hypophysitis is an irAE that commonly presents following CTLA-4 antibody blockage but not with PD-1 inhibitor treatment (1). Symptoms of pituitary dysfunction are extensive, and include headache, weakness, visual changes, and enlargement of the pituitary gland (57). Pituitary inflammation induces secondary adrenal insufficiency, secondary adrenocorticotropic hormone (ACTH) deficiency, secondary hypothyroidism, and hypogonadotropin hypogonadism (1)

\section{Therapeutic Management}

Several retrospective cohort studies have suggested that high doses of systemic corticosteroid therapy are not effective in reducing pituitary inflammation (58). Therefore, endocrinerelated irAEs still require clinical exploration of more effective control methods, as long as immunotherapy is not terminated or the efficacy of antibodies is not affected.

\section{Association With Clinical Outcomes}

A clinical study of CTLA-4 antibody in melanoma patients with hypophysitis suggested better antitumor efficacy was achieved (59).

\section{Possible Mechanisms/Pathophysiology}

Some data suggest that pituitary inflammation may be associated with B-cell immunotoxicity and autoantibody production, including upregulation of anti-GNAL antibodies, or antiITM2B antibodies in patients with pituitary inflammation (1).

\section{PANCREATITIS}

\section{Clinical Characteristics}

The pancreas is an organ rarely affected by $\mathrm{PD}-1$ antibody treatment in lung cancer therapy. The clinical features of irAEassociated pancreatitis are varied and difficult to identify. Asymptomatic elevation of serum lipase and/or amylase levels during ICI treatment hampers the diagnostic process. During ICI therapy, serum lipase and/or amylase may be elevated, but the patient remains asymptomatic (60). 


\section{Therapeutic Management and Association With Clinical Outcomes}

The treatment of pancreatitis remains a difficult clinical problem, and immunotherapy may have to be suspended in due course. At present, the treatment of pancreatitis involves large doses of systemic glucocorticoids, and requires long-term administration, which gradually reduces patient symptoms and allows normalization of serum lipase levels. Delayed secondary pancreatic insufficiency may occur even after successful treatment, and patients must be regularly monitored (60).

\section{Possible Mechanisms/Pathophysiology}

Pancreatitis is a rare immune-associated adverse event with PD1 antibody treatment. Its imaging features are similar to those of autoimmune pancreatitis. Clinical evidence suggests that the pathologic characteristics of nivolumab in treating pancreatitis are similar to those of autoimmune pancreatitis $(60,61)$.

\section{TREATMENT OF IRAES IN LUNG CANCER TREATMENT}

Steroids and/or immunosuppressants are common clinical treatments for irAEs, and may this be associated with reduced efficacy of cancer immunotherapy. Given their immunosuppressive activity, the potential effects of glucocorticoids on the anticancer activity on inhibition of immune checkpoints must be considered. The results of multiple retrospective studies investigating melanoma are exciting (62). Steroid use was not associated with reduced efficacy of CTLA-4 inhibitors and PD-1 or PD-L1 inhibitors. Interestingly, patients exhibiting irAEs experienced a longer progression-free survival than patients without irAEs, and the benefits did not change with steroid use. Nonetheless, the use of prednisone during early treatment is associated with a poorer prognosis in lung cancer patients (63). Thus, prospective studies are still needed to determine the effects of steroid use on lung cancer outcomes in patients receiving PD-1 antibody therapy. These data suggest caution in the use of steroids or immunosuppressants.

In addition, low doses of corticosteroids can significantly impair the antitumor activity of $\mathrm{T}$ cells. Different organs also present different adverse effects (64). Therefore, additional clinical trials are needed to verify whether safer targeted drugs or antibody drugs are more feasible based on the organ-specific mechanisms associated with immune-related adverse events following treatment with ICIs. For now, treatments for moderate or severe irAEs in a timely manner is needed.

\section{DISCUSSION}

Inhibition of immune checkpoints, especially PD-1 blockade, represents an increasingly important strategy in cancer treatment. Overall, treatment with PD-1 antibodies is relatively safe for lung cancer, and most induced irAEs are clinically manageable $(1,15)$. Most toxic effects are reversible, except effects on the endocrine system may be long-lasting. Deaths from irAEs are rare, but myocarditis, pneumonia and colitis may likely trigger them. Therefore, attentive clinical monitoring and management is very important.

Here, we mainly review the irAEs in lung cancer treated with PD-1 antibody. Is there any difference with other types of cancer? Cutaneous malignancies (including melanoma, squamous cell carcinoma of the skin, and basal cell carcinoma) with treatment with PD-1 antibody have a high incidence of dermatitis as to $43 \%$, and the incidence of head and neck cancer was also increased to $20 \%$, both significantly higher than that of lung cancer patients. Patients with cutaneous malignancies were significantly more likely to develop dermatitis than patients with noncutaneous malignancy, including lung cancer (65). Pneumonitis is a relatively rare irAE in PD-1 therapy. The incidence of pneumonia was $\sim 1 \%$ in melanoma and renal cell carcinoma patients receiving PD-1 inhibitor monotherapy, and rose to $3.1 \%$ in non-small cell lung cancer patients $(66,67)$. These data suggest that tumorigenetic organs may exhibit a higher frequency of irAEs. In general, there is no significant difference in the occurrence of irAEs among different types of tumors (1).

Currently, several PD-1 inhibitor drugs have been marketed, among which the most widely used are pembrolizumab and nivolumab (1). There is no a depth view of the difference in percentage of irAEs regarding different anti PD-1 therapies such as pembrolizumab and nivolumab. Shrujal et al. reported that organ specific irAEs were evaluated with 2993 patients in the investigational arm (pembrolizumab 1459, nivolumab 1534) (2). Among the 1459 patients exposed to pembrolizumab 1.1\% had colitis, $0.2 \%$ had hepatitis, $3.1 \%$ had pneumonitis, $7.6 \%$ had hypothyroidism and $0.4 \%$ had hypophysitis. Among the 1534 patients exposed to nivolumab $0.3 \%$ had colitis, $0.0 \%$ had hepatitis, $2.2 \%$ had pneumonitis, $5.9 \%$ had hypothyroidism and $0.3 \%$ had hypophysitis (2). These data suggest organ specific irAEs are uncommon with the anti-PD-1 drugs. General irAEs are largely similar. The rates of pembrolizumab induced irAEs was slightly higher than that of nivolumab. The reason for this slight difference remains an open question.

Consistent with the different functions of immune checkpoints, the types of irAEs associated with monotherapy targeting the CTLA-4 or PD-1 pathways also differ (68). Typically, PD-1 inhibitors are better tolerated than CTLA-4 inhibitors. Grade 3 and 4 irAEs are more common in CTLA-4 inhibitors than in PD-1 inhibitors (69). Of note, colitis, rash and hypophysitis were more common with CTLA-4 inhibitors, whereas arthralgia, pneumonitis, vitiligo, and hypothyroidism were more common with PD-1 inhibitors (70). The exact biological explanation for the differences in organ selectivity and severity in irAEs with different ICIs is not fully understood. Theoretically, CTLA-4 blockade might induce larger $\mathrm{T}$ cell proliferation and also down-regulate regulatory $\mathrm{T}$ (Treg) cells, while PD-1 blockade only activates $\mathrm{T}$ cell clones in a small number of lesions (71).

There have been few studies on biomarkers for the risk of developing irAEs of immune checkpoint inhibitor therapy. Specific 
CD8+ T cells, Interleukin 17, eosinophil counts have been related to irAEs but not Set the threshold $(72,73)$. There are some preliminary clinical data suggesting that a family history of autoimmune diseases, previous viral infections, and known autoimmunotoxic drugs are also potential related risk factors $(74,75)$. It has recently been reported that irAEs were more frequent among patients with he preexisting antibodies (76). For example, skin reactions are more common in patients who already have rheumatoid factor than in patients who don't (76). Thyroid dysfunction is more common in patients with pre-existing anti-thyroid antibodies. Suzuki et al. reported that 12 of 9869 cancer patients treated with nivolumab developed myasthenia gravis, 10 of whom had pre-existing acetylcholine receptor antibodies (77). Therefore, it is worth further investigation that pre-existing factor is associated with the development of irAEs.

\section{REFERENCES}

1. Ramos-Casals M, Brahmer JR, Callahan MK, Flores-Chávez A, Keegan N, Khamashta MA, et al. Immune-Related Adverse Events of Checkpoint Inhibitors. Nat Rev Dis Primers (2020) 6:38. doi: 10.1038/s41572-020-0160-6

2. Baxi S, Yang A, Gennarelli RL, Khan N, Wang Z, Boyce L, et al. ImmuneRelated Adverse Events for anti-PD-1 and anti-PD-L1 Drugs: Systematic Review and Meta-Analysis. Bmj (2018) 360:k793. doi: 10.1136/bmj.k793

3. Garon EB, Rizvi NA, Hui R, Leighl N, Balmanoukian AS, Eder JP, et al. Pembrolizumab for the Treatment of non-Small-Cell Lung Cancer. New Engl J Med (2015) 372:2018-28. doi: 10.1056/NEJMoa1501824

4. Borghaei H, Paz-Ares L, Horn L, Spigel DR, Steins M, Ready NE, et al. Nivolumab Versus Docetaxel in Advanced Nonsquamous Non-Small-Cell Lung Cancer. New Engl J Med (2015) 373:1627-39. doi: 10.1056/ NEJMoa1507643

5. Gettinger S, Rizvi NA, Chow LQ, Borghaei H, Brahmer J, Ready N, et al. Nivolumab Monotherapy for First-Line Treatment of Advanced non-SmallCell Lung Cancer. J Clin Oncol (2016) 34:2980-7. doi: 10.1200/ JCO.2016.66.9929

6. Topalian SL, Hodi FS, Brahmer JR, Gettinger SN, Smith DC, McDermott DF, et al. Safety, Activity, and Immune Correlates of anti-PD-1 Antibody in Cancer. New Engl J Med (2012) 366:2443-54. doi: 10.1056/NEJMoa1200690

7. Herbst RS, Baas P, Kim D-W, Felip E, Pérez-Gracia JL, Han J-Y, et al. Pembrolizumab Versus Docetaxel for Previously Treated, PD-L1-positive, Advanced non-Small-Cell Lung Cancer (KEYNOTE-010): A Randomised Controlled Trial. Lancet (2016) 387:1540-50. doi: 10.1016/S0140-6736(15) 01281-7

8. Leighl NB, Hellmann MD, Hui R, Carcereny E, Felip E, Ahn M-J, et al. Pembrolizumab in Patients With Advanced non-Small-Cell Lung Cancer (KEYNOTE-001): 3-Year Results From an Open-Label, Phase 1 Study. Lancet Respir Med (2019) 7:347-57. doi: 10.1016/S2213-2600(18)30500-9

9. Brahmer J, Reckamp KL, Baas P, Crinò L, Eberhardt WE, Poddubskaya E, et al. Nivolumab Versus Docetaxel in Advanced Squamous-Cell Non-SmallCell Lung Cancer. New Engl J Med (2015) 373:123-35. doi: 10.1056/ NEJMoa1504627

10. Osorio JC, Ni A, Chaft JE, Pollina R, Kasler MK, Stephens D, et al. AntibodyMediated Thyroid Dysfunction During T-cell Checkpoint Blockade in Patients With non-Small-Cell Lung Cancer. Ann Oncol (2017) 28:583-9. doi: 10.1093/annonc/mdw640

11. Garon EB, Rizvi NA, Hui R, Leighl N, Balmanoukian AS, Eder JP, et al. Pembrolizumab for the Treatment of Non-Small-Cell Lung Cancer. New Engl J Med (2015) 372:2018-28. doi: 10.1056/NEJMoa1501824

12. Hui R, Garon EB, Goldman JW, Leighl NB, Hellmann MD, Patnaik A, et al. Pembrolizumab as First-Line Therapy for Patients With PD-L1-positive Advanced non-Small Cell Lung Cancer: A Phase 1 Trial. Ann Oncol (2017) 28:874-81. doi: 10.1093/annonc/mdx008

13. Mok TSK, Wu Y-L, Kudaba I, Kowalski DM, Cho BC, Turna HZ, et al. Pembrolizumab Versus Chemotherapy for Previously Untreated, PD-L1-

\section{AUTHOR CONTRIBUTIONS}

HW and XZ conceived and conducted the project. HW supervised the project. XZ and HW wrote the paper. All authors contributed to the article and approved the submitted version.

\section{FUNDING}

This work was supported by the Natural Science Foundation of China (Reference Numbers: 81872318), CAMS Innovation Fund for Medical Sciences (CIFMS, 2019-I2M-5-073) and the Strategic Priority Research Program of the Chinese Academy of Sciences (XDPB1002).

expressing, Locally Advanced or Metastatic non-Small-Cell Lung Cancer (KEYNOTE-042): A Randomised, Open-Label, Controlled, Phase 3 Trial. Lancet (2019) 393:1819-30. doi: 10.1016/S0140-6736(18)32409-7

14. Rizvi NA, Mazières J, Planchard D, Stinchcombe TE, Dy GK, Antonia SJ, et al. Activity and Safety of Nivolumab, an anti-PD-1 Immune Checkpoint Inhibitor, for Patients With Advanced, Refractory Squamous non-SmallCell Lung Cancer (CheckMate 063): A Phase 2, Single-Arm Trial. Lancet Oncol (2015) 16:257-65. doi: 10.1016/S1470-2045(15)70054-9

15. Reck M, Rodríguez-Abreu D, Robinson AG, Hui R, Csosszi T, Fülöp A, et al. Pembrolizumab Versus Chemotherapy for PD-L1-Positive non-Small-Cell Lung Cancer. New Engl J Med (2016) 375:1823-33. doi: 10.1056/NEJMoa1606774

16. McLachlan SM, Rapoport B. Breaking Tolerance to Thyroid Antigens: Changing Concepts in Thyroid Autoimmunity. Endocr Rev (2014) 35:59105. doi: 10.1210/er.2013-1055

17. Postow MA, Longo DL, Sidlow R, Hellmann MD. Immune-Related Adverse Events Associated With Immune Checkpoint Blockade. New Engl J Med (2018) 378:158-68. doi: 10.1056/NEJMra1703481

18. Min Lee CK, Li S, Tran DC, Zhu GA, Kim J, Kwong BY, et al. Characterization of Dermatitis After PD-1/PD-L1 Inhibitor Therapy and Association With Multiple Oncologic Outcomes: A Retrospective Case-Control Study. J Am Acad Dermatol (2018) 79:1047-52. doi: 10.1016/j.jaad.2018.05.035

19. Gettinger SN, Horn L, Gandhi L, Spigel DR, Antonia SJ, Rizvi NA, et al. Overall Survival and Long-Term Safety of Nivolumab (Anti-Programmed Death 1 Antibody, Bms-936558, ONO-4538) in Patients With Previously Treated Advanced non-Small-Cell Lung Cancer. J Clin Oncol (2015) 33:200412. doi: 10.1200/JCO.2014.58.3708

20. Brahmer JR, Lacchetti C, Schneider BJ, Atkins MB, Brassil KJ, Caterino JM, et al. Management of Immune-Related Adverse Events in Patients Treated With Immune Checkpoint Inhibitor Therapy: American Society of Clinical Oncology Clinical Practice Guideline. J Clin Oncol (2018) 36:1714-68. doi: 10.1200/JCO.2017.77.6385

21. Lee Y, Shim HS, Park MS, Kim JH, Ha SJ, Kim SH, et al. High EGFR Gene Copy Number and Skin Rash as Predictive Markers for EGFR Tyrosine Kinase Inhibitors in Patients With Advanced Squamous Cell Lung Carcinoma. Clin Cancer Res (2012) 18:1760-8. doi: 10.1158/1078-0432.CCR-11-2582

22. Teulings HE, Limpens J, Jansen SN, Zwinderman AH, Reitsma JB, Spuls PI, et al. Vitiligo-Like Depigmentation in Patients With Stage III-IV Melanoma Receiving Immunotherapy and its Association With Survival: A Systematic Review and Meta-Analysis. J Clin Oncol (2015) 33:773-81. doi: 10.1200/ JCO.2014.57.4756

23. Mohamed MK, Ramalingam S, Lin Y, Gooding W, Belani CP. Skin Rash and Good Performance Status Predict Improved Survival With Gefitinib in Patients With Advanced non-Small Cell Lung Cancer. Ann Oncol (2005) 16:780-5. doi: 10.1093/annonc/mdil57

24. Pérez-Soler R, Chachoua A, Hammond LA, Rowinsky EK, Huberman M, Karp D, et al. Determinants of Tumor Response and Survival With Erlotinib in Patients With non-Small-Cell Lung Cancer. J Clin Oncol (2004) 22:323847. doi: 10.1200/JCO.2004.11.057 
25. Poprach A, Pavlik T, Melichar B, Puzanov I, Dusek L, Bortlicek Z, et al. Skin Toxicity and Efficacy of Sunitinib and Sorafenib in Metastatic Renal Cell Carcinoma: A National Registry-Based Study. Ann Oncol (2012) 23:3137-43. doi: 10.1093/annonc/mds145

26. Phan GQ, Attia P, Steinberg SM, White DE, Rosenberg SA. Factors Associated With Response to High-Dose Interleukin-2 in Patients With Metastatic Melanoma. J Clin Oncol (2001) 19:3477-82. doi: 10.1200/JCO. 2001.19.15.3477

27. Sanlorenzo M, Vujic I, Daud A, Algazi A, Gubens M, Luna SA, et al. Pembrolizumab Cutaneous Adverse Events and Their Association With Disease Progression. JAMA Dermatol (2015) 151:1206-12. doi: 10.1001/ jamadermatol.2015.1916

28. Delyon J, Mateus C, Lefeuvre D, Lanoy E, Zitvogel L, Chaput N, et al. Experience in Daily Practice With Ipilimumab for the Treatment of Patients With Metastatic Melanoma: An Early Increase in Lymphocyte and Eosinophil Counts is Associated With Improved Survival. Ann Oncol (2013) 24:1697703. doi: $10.1093 /$ annonc/mdt027

29. Joseph RW, Cappel M, Goedjen B, Gordon M, Kirsch B, Gilstrap C, et al. Lichenoid Dermatitis in Three Patients With Metastatic Melanoma Treated With anti-PD-1 Therapy. Cancer Immunol Res (2015) 3:18-22. doi: 10.1158/ 2326-6066.CIR-14-0134

30. Okiyama N, Fujimoto M. Clinical Perspectives and Murine Models of Lichenoid Tissue Reaction/Interface Dermatitis. J Dermatol Sci (2015) 78:167-72. doi: 10.1016/j.jdermsci.2015.03.001

31. Cramer P, Bresalier RS. Gastrointestinal and Hepatic Complications of Immune Checkpoint Inhibitors. Curr Gastroenterol Rep (2017) 19:3. doi: 10.1007/s11894-017-0540-6

32. Hofmann L, Forschner A, Loquai C, Goldinger SM, Zimmer L, Ugurel S, et al. Cutaneous, Gastrointestinal, Hepatic, Endocrine, and Renal Side-Effects of anti-PD-1 Therapy. Eur J Cancer (Oxford England: 1990) (2016) 60:190-209. doi: 10.1016/j.ejca.2016.02.025

33. Karamchandani DM, Chetty R. Immune Checkpoint Inhibitor-Induced Gastrointestinal and Hepatic Injury: Pathologists' Perspective. J Clin Pathol (2018) 71:665-71. doi: 10.1136/jclinpath-2018-205143

34. Davar D, Wilson M, Pruckner C, Kirkwood JM. Pd-1 Blockade in Advanced Melanoma in Patients With Hepatitis C and/or HIV. Case Rep Oncol Med (2015) 2015:737389. doi: 10.1155/2015/737389

35. Geukes Foppen MH, Rozeman EA, van Wilpe S, Postma C, Snaebjornsson P, van Thienen JV, et al. Immune Checkpoint Inhibition-Related Colitis: Symptoms, Endoscopic Features, Histology and Response to Management. ESMO Open (2018) 3:e000278. doi: 10.1136/esmoopen-2017-000278

36. Hughes MS, Zheng H, Zubiri L, Molina GE, Chen ST, Mooradian MJ, et al. Colitis After Checkpoint Blockade: A Retrospective Cohort Study of Melanoma Patients Requiring Admission for Symptom Control. Cancer Med (2019) 8:4986-99. doi: 10.1002/cam4.2397

37. Abu-Sbeih H, Ali FS, Luo W, Qiao W, Raju GS, Wang Y. Importance of Endoscopic and Histological Evaluation in the Management of Immune Checkpoint Inhibitor-Induced Colitis. J Immunother Cancer (2018) 6:95. doi: 10.1186/s40425-018-0411-1

38. Weber JS, Postow M, Lao CD, Schadendorf D. Management of Adverse Events Following Treatment With Anti-Programmed Death-1 Agents. Oncol (2016) 21:1230-40. doi: 10.1634/theoncologist.2016-0055

39. Weber JS, Yang JC, Atkins MB, Disis ML. Toxicities of Immunotherapy for the Practitioner. J Clin Oncol (2015) 33:2092-9. doi: 10.1200/ JCO.2014.60.0379

40. Fecher LA, Agarwala SS, Hodi FS, Weber JS. Ipilimumab and its Toxicities: A Multidisciplinary Approach. Oncol (2013) 18:733-43. doi: 10.1634/ theoncologist.2012-0483

41. Assarzadegan N, Montgomery E, Anders RA. Immune Checkpoint Inhibitor Colitis: The Flip Side of the Wonder Drugs. Virchows Archiv (2018) 472:12533. doi: $10.1007 / \mathrm{s} 00428-017-2267-\mathrm{z}$

42. Chen JH, Pezhouh MK, Lauwers GY, Masia R. Histopathologic Features of Colitis Due to Immunotherapy With Anti-PD-1 Antibodies. Am J Surg Pathol (2017) 41:643-54. doi: 10.1097/PAS.0000000000000829

43. Gonzalez RS, Salaria SN, Bohannon CD, Huber AR, Feely MM, Shi C. PD-1 Inhibitor Gastroenterocolitis: Case Series and Appraisal of 'Immunomodulatory Gastroenterocolitis'. Histopathology (2017) 70:558-67. doi: 10.1111/his.13118
44. Nishino M, Ramaiya NH, Awad MM, Sholl LM, Maattala JA, Taibi M, et al. Pd-1 Inhibitor-Related Pneumonitis in Advanced Cancer Patients: Radiographic Patterns and Clinical Course. Clin Cancer Res (2016) 22:6051-60. doi: 10.1158/1078-0432.CCR-16-1320

45. Nishino M, Sholl LM, Hodi FS, Hatabu H, Ramaiya NH. Anti-Pd-1-Related Pneumonitis During Cancer Immunotherapy. New Engl J Med (2015) 373:288-90. doi: 10.1056/NEJMc1505197

46. Nishino M, Chambers ES, Chong CR, Ramaiya NH, Gray SW, Marcoux JP, et al. Anti-Pd-1 Inhibitor-Related Pneumonitis in Non-Small Cell Lung Cancer. Cancer Immunol Res (2016) 4:289-93. doi: 10.1158/2326-6066.CIR-15-0267

47. Gettinger SN, Horn L, Gandhi L, Spigel DR, Antonia SJ, Rizvi NA, et al. Overall Survival and Long-Term Safety of Nivolumab (Anti-Programmed Death 1 Antibody, Bms-936558, ONO-4538) in Patients With Previously Treated Advanced non-Small-Cell Lung Cancer. J Clin Oncol (2015) 33:200412. doi: 10.1200/JCO.2014.58.3708

48. Postow MA, Chesney J, Pavlick AC, Robert C, Grossmann K, McDermott D, et al. Nivolumab and Ipilimumab Versus Ipilimumab in Untreated Melanoma. New Engl J Med (2015) 372:2006-17. doi: 10.1056/NEJMoa1414428

49. Wanchoo R, Karam S, Uppal NN, Barta VS, Deray G, Devoe C, et al. Adverse Renal Effects of Immune Checkpoint Inhibitors: A Narrative Review. Am J Nephrol (2017) 45(2):160-9. doi: 10.1159/000455014

50. Vandiver JW, Singer Z, Harshberger C. Severe Hyponatremia and Immune Nephritis Following an Initial Infusion of Nivolumab. Target Oncol (2016) 11:553-6. doi: 10.1007/s11523-016-0426-9

51. Ramos-Casals M, Lambotte O, Kostine M, Calabrese L, Suarez-Almazor M, Bingham C, et al. Thu0649 Phenotypic Clusters of Rheumatic/Systemic Immune-Related Adverse Events Induced by Cancer Immunotherapies (Immunocancer International Registry). Poster Present (2019) pp:620-1. doi: 10.1136/annrheumdis-2019-eular.2870

52. Perazella MA, Shirali AC. Immune Checkpoint Inhibitor Nephrotoxicity: What do We Know and What Should We do? Kidney Int (2020) 97:62-74. doi: 10.1016/j.kint.2019.07.022

53. Gonzalez NL, Puwanant A, Lu A, Marks SM, Živković SA. Myasthenia Triggered by Immune Checkpoint Inhibitors: New Case and Literature Review. Neuromusc Disord: NMD (2017) 27:266-8. doi: 10.1016/ j.nmd.2017.01.002

54. Anquetil C, Salem JE, Lebrun-Vignes B, Johnson DB, Mammen AL, Stenzel W, et al. Immune Checkpoint Inhibitor-Associated Myositis: Expanding the Spectrum of Cardiac Complications of the Immunotherapy Revolution Circulation (2018) 138:743-5. doi: 10.1161/CIRCULATIONAHA.118.035898

55. Touat M, Maisonobe T, Knauss S, Ben Hadj Salem O, Hervier B, Aure K, et al. Immune Checkpoint Inhibitor-Related Myositis and Myocarditis in Patients With Cancer. Neurology (2018) 91:e985-94. doi: 10.1212/WNL.0000000000006124

56. Arbuckle MR, McClain MT, Rubertone MV, Scofield RH, Dennis GJ, James JA, et al. Development of Autoantibodies Before the Clinical Onset of Systemic Lupus Erythematosus. New Engl J Med (2003) 349:1526-33. doi: 10.1056/NEJMoa021933

57. Dillard T, Yedinak CG, Alumkal J, Fleseriu M. Anti-CTLA-4 Antibody Therapy Associated Autoimmune Hypophysitis: Serious Immune Related Adverse Events Across a Spectrum of Cancer Subtypes. Pituitary (2010) 13:29-38. doi: 10.1007/s11102-009-0193-Z

58. Min L, Hodi FS, Giobbie-Hurder A, Ott PA, Luke JJ, Donahue H, et al. Systemic High-Dose Corticosteroid Treatment Does Not Improve the Outcome of Ipilimumab-Related Hypophysitis: A Retrospective Cohort Study. Clin Cancer Res (2014) 21:749-55. doi: 10.1158/1078-0432.CCR-14-2353

59. Faje AT, Sullivan R, Lawrence D, Tritos NA, Fadden R, Klibanski A, et al. Ipilimumab-Induced Hypophysitis: A Detailed Longitudinal Analysis in a Large Cohort of Patients With Metastatic Melanoma. J Clin Endocrinol Metab (2014) 99:4078-85. doi: 10.1210/jc.2014-2306

60. Kohlmann J, Wagenknecht D, Simon JC, Ziemer M. Immune-Related Pancreatitis Associated With Checkpoint Blockade in Melanoma. Melanoma Res (2019) 29:549-52. doi: 10.1097/CMR.0000000000000611

61. Saito H, Ono K. Nivolumab-Induced Pancreatitis: An Immune-related Adverse Event. Radiology (2019) 293:521. doi: 10.1148/radiol.2019191603

62. Weber JS, Hodi FS, Wolchok JD, Topalian SL, Schadendorf D, Larkin J, et al. Safety Profile of Nivolumab Monotherapy: A Pooled Analysis of Patients With Advanced Melanoma. J Clin Oncol (2017) 35:785-92. doi: 10.1200/ JCO.2015.66.1389 
63. Arbour KC, Mezquita L, Long N, Rizvi H, Auclin E, Ni A, et al. Impact of Baseline Steroids on Efficacy of Programmed Cell Death-1 and Programmed Death-Ligand 1 Blockade in Patients With Non-Small-Cell Lung Cancer. J Clin Oncol (2018) 36:2872-8. doi: 10.1200/JCO.2018.79.0006

64. Maher VE, Fernandes LL, Weinstock C, Tang S, Agarwal S, Brave M, et al. Analysis of the Association Between Adverse Events and Outcome in Patients Receiving a Programmed Death Protein 1 or Programmed Death Ligand 1 Antibody. J Clin Oncol (2019) 37:2730-7. doi: 10.1200/JCO.19.00318

65. Hassel JC, Heinzerling L, Aberle J, Bähr O, Eigentler TK, Grimm MO, et al. Combined Immune Checkpoint Blockade (Anti-PD-1/Anti-CTLA-4): Evaluation and Management of Adverse Drug Reactions. Cancer Treat Rev (2017) 57:36-49. doi: 10.1016/j.ctrv.2017.05.003

66. Nishino M, Giobbie-Hurder A, Hatabu H, Ramaiya NH, Hodi FS. Incidence of Programmed Cell Death 1 Inhibitor-Related Pneumonitis in Patients With Advanced Cancer: A Systematic Review and Meta-Analysis. JAMA Oncol (2016) 2:1607-16. doi: 10.1001/jamaoncol.2016.2453

67. Ma K, Lu Y, Jiang S, Tang J, Li X, Zhang Y. The Relative Risk and Incidence of Immune Checkpoint Inhibitors Related Pneumonitis in Patients With Advanced Cancer: A Meta-Analysis. Front Pharmacol (2018) 9:1430. doi: 10.3389/fphar.2018.01430

68. Pauken KE, Dougan M, Rose NR, Lichtman AH, Sharpe AH. Adverse Events Following Cancer Immunotherapy: Obstacles and Opportunities. Trends Immunol (2019) 40:511-23. doi: 10.1016/j.it.2019.04.002

69. Larkin J, Chiarion-Sileni V, Gonzalez R, Grob JJ, Cowey CL, Lao CD, et al. Combined Nivolumab and Ipilimumab or Monotherapy in Untreated Melanoma. New Engl J Med (2015) 373:23-34. doi: 10.1056/NEJMoa1504030

70. Khoja L, Day D, Wei-Wu Chen T, Siu LL, Hansen AR. Tumour- and ClassSpecific Patterns of Immune-Related Adverse Events of Immune Checkpoint Inhibitors: A Systematic Review. Ann Oncol (2017) 28:2377-85. doi: 10.1093/ annonc/mdx 286

71. Seidel JA, Otsuka A, Kabashima K. Anti-PD-1 and Anti-CTLA-4 Therapies in Cancer: Mechanisms of Action, Efficacy, and Limitations. Front Oncol (2018) 8:86. doi: $10.3389 /$ fonc. 2018.00086
72. Shahabi V, Berman D, Chasalow SD, Wang L, Tsuchihashi Z, Hu B, et al Gene Expression Profiling of Whole Blood in Ipilimumab-Treated Patients for Identification of Potential Biomarkers of Immune-Related Gastrointestinal Adverse Events. J Trans Med (2013) 11:75. doi: 10.1186/1479-5876-11-75

73. Subudhi SK, Aparicio A, Gao J, Zurita AJ, Araujo JC, Logothetis CJ, et al. Clonal Expansion of CD8 T Cells in the Systemic Circulation Precedes Development of Ipilimumab-Induced Toxicities. Proc Natl Acad Sci USA (2016) 113:11919-24. doi: 10.1073/pnas.1611421113

74. Champiat S, Lambotte O, Barreau E, Belkhir R, Berdelou A, Carbonnel F, et al. Management of Immune Checkpoint Blockade Dysimmune Toxicities: A Collaborative Position Paper. Ann Oncol (2016) 27:559-74. doi: 10.1093/ annonc/mdv623

75. Manson G, Norwood J, Marabelle A, Kohrt H, Houot R. Biomarkers Associated With Checkpoint Inhibitors. Ann Oncol (2016) 27:1199-206. doi: 10.1093/annonc/mdw181

76. Toi Y, Sugawara S, Sugisaka J, Ono H, Kawashima Y, Aiba T, et al. Profiling Preexisting Antibodies in Patients Treated With Anti-Pd-1 Therapy for Advanced non-Small Cell Lung Cancer. JAMA Oncol (2019) 5:376-83. doi: 10.1001/jamaoncol.2018.5860

77. Suzuki S, Ishikawa N, Konoeda F, Seki N, Fukushima S, Takahashi K, et al. Nivolumab-Related Myasthenia Gravis With Myositis and Myocarditis in Japan. Neurology (2017) 89:1127-34. doi: 10.1212/WNL.0000000000004359

Conflict of Interest: The authors declare that the research was conducted in the absence of any commercial or financial relationships that could be construed as a potential conflict of interest.

Copyright (c) 2021 Zheng and Wei. This is an open-access article distributed under the terms of the Creative Commons Attribution License (CC BY). The use, distribution or reproduction in other forums is permitted, provided the original author $(s)$ and the copyright owner(s) are credited and that the original publication in this journal is cited, in accordance with accepted academic practice. No use, distribution or reproduction is permitted which does not comply with these terms. 